\title{
Photothermal conversion hydrogel based mini-eye patch for relieving dry eye with long-term use of the light-emitting screen
}

This article was published in the following Dove Press journal: International Journal of Nanomedicine

\author{
Yulian Pang ${ }^{1} *$ \\ Chaochao $\mathrm{Wei}^{2, *}$ \\ Ruolei $\mathrm{Li}^{3}$ \\ Yue $\mathrm{Wu}^{4}$ \\ Wei Liu $^{2}$ \\ Feifei Wang' \\ Xu Zhang' \\ Xiaolei Wang ${ }^{2,5}$
}

'Jiangxi Provincial Key Laboratory for Ophthalmology, Affiliated Eye Hospital of Nanchang University, Jiangxi Research Institute of Ophthalmology \& Visual Science, Nanchang 330006, People's Republic of China; ${ }^{2}$ College of Chemistry, Nanchang University, Nanchang 330088, People's Republic of China; ${ }^{3}$ College of Medicine, Nanchang University,

Nanchang 330088, People's Republic of

China; ${ }^{4}$ Queen Mary School of Nanchang

University, Nanchang University,

Nanchang 330088, People's Republic of

China; ${ }^{5}$ Institute of Translational

Medicine, Nanchang University,

Nanchang 330088, People's Republic of China

*These authors contributed equally to this work

Correspondence: Xu Zhang Jiangxi Provincial Key Laboratory for Ophthalmology, Affiliated Eye Hospital of Nanchang University, Jiangxi Research Institute of Ophthalmology \& Visual Science, 463 Bayi Road, Nanchang 330006, People's Republic of China Tel +86I 3647008729

Email xuzhang19@163.com

Xiaolei Wang

Institute of Translational Medicine,

Nanchang University, Hong Gu Tan New

District, I299 Xuefu Road, Nanchang

330088, People's Republic of China

Tel +86I 8679850415

Email wangxiaolei@ncu.edu.cn
Purpose: The frequent usage of various lighting screens has made dry eye syndrome an increasingly serious phenomenon. To relieve this global problem, we have developed a photothermal conversion hydrogel based mini-eye patch.

Methods: Gold nanoparticles (GNRs) were synthesized by a seed-mediated method, and then used as the inner cores to grow palladium (Pd) shell by PdCl42-reduction. Then, gelatin was added to prepare GNRs @ Pd hydrogel eye patch by genipin cross-linking. We implanted temperature sensitive ink (complex composed of amino resin and styrene maleic anhydride copolymer) in the eye patch, which could change color at different temperatures. Heating performance of the eye patch was accessed with an infrared temperature profile and the circulating temperature experiment. The safety assessment of the eye patch was conducted by H\&E staining of the mouse's eyelid skin and CCK-8 assay. A Keratograph 5M non invasive ocular surface analyzer was used to assess the impact of eye patches on dry eyes. Results: It was found that GNRs@ @ Pd hydrogel eye patches could sense various visible light and responded by heating up spontaneously. Results from the CCK-8 assay and H\&E staining showed that the eye patch has good safety performance. Measurements of the first noninvasive tear break-up time (NITBUT), the average NITBUT, the tear meniscus height (TMH), combined with red eye analysis, further demonstrated the patch's eye-protective properties.

Conclusion: After being pasted to the lacrimal gland, the hydrogel patch converted various light irradiations into heat and stimulated the lacrimal gland to produce more tears to relieve dry eye. The built-in temperature-sensitive ink can play an important role in warning people of their excessive eye usage. Because this recyclable strategy does not interfere with normal eye use, it is thus more environmentally friendly and convenient than ordinary infrared eyewear.

Keywords: nanotechnology, palladium, gold, hydrogel

\section{Introduction}

With the popularization of video terminals, the incidence of dry eye has increased year by year. ${ }^{1-3}$ At present, clinically, dry eye syndrome is mainly divided into two categories: "dry eye with reduced tear production (aqueous-deficient)," and "dry eye with increased evaporation of the tear film (hyperevaporative)". 4 Around $10 \%$ of patients with dry eye have a solely aqueous-deficient disorder. Mixed hyperevaporative/aqueous-deficient forms account for more than $80 \%$ of cases. ${ }^{5-7}$ Therefore, the improvement of aqueous-deficient is still an important issue in the treatment of dry eye syndrome. Currently, the most important method for treating dry eye is tear replacement therapy, which uses artificial tears locally to improve 
ocular surface humidity and lubrication capacity. ${ }^{4}$ However, because the components of the natural tears are complicated, artificial tears cannot substitute them completely. Moreover, many commercially available artificial tears contain preservatives. Even though the concentration may be very low, their damage to ocular cells cannot be ignored. ${ }^{8,9}$ Recently, infrared health eye masks for treating and preventing dry eye have also appeared on the market. However, most of them are disposable or need to be recharged, and they interfere with normal use of the eyes. A need for convenient and healthy eyes protection remains to be addressed. If we can develop a smart eye protection material, capable of adjusting natural tear secretion automatically according to the external light source intensity, dry eye could be relieved without charging the protective wear, artificial tears, or interference with normal eye usage.

To achieve this goal, the core aim is to convert visible light energy into heat, which can thus stimulate the lacrimal secretion of tears to prevent dry eye. In selecting photothermal conversion materials, it was found that palladium-coated gold nanorods have higher photothermal conversion efficiency ${ }^{10-12}$ than the same dose of pure palladium $^{13}$ or pure gold. ${ }^{14-16}$ We utilized gold nanorods @Pd (GNRs@Pd) for the study. To obtain GNRs@Pd nanomaterials, GNRs ${ }^{17,18}$ were synthesized using a seedmediated method, and the GNRs were used to grow the Pd shell by $\mathrm{PdCl}_{4}{ }^{2-}$ reduction. After that, GNRs@ $\mathrm{Pd}^{19}$ and gelatin were used as raw materials to prepare gelatin hydrogel $^{20,21}$ eye patches through genipin cross-linking. ${ }^{22}$

\section{Materials and methods Materials}

Tetrachloroauric acid $\left(\mathrm{HAuCl}_{4} \cdot 4 \mathrm{H}_{2} \mathrm{O}\right)$, cetyltrimethyl ammonium bromide (CTAB), silver nitrate $(0.01 \mathrm{M})$, ascorbic acid $(0.1 \mathrm{M})$, sodium borohydride $(0.01 \mathrm{M})$, $\mathrm{PdCl}_{2}$, aqueous $\mathrm{HCl}$ solution $(0.2 \mathrm{M})$, geniping, and gelatin were purchased from Macklin Co. (Shanghai, China).

\section{Synthesis of gold nanorods (GNRs)}

To begin, $5 \mathrm{~mL}$ CTAB solution $(0.2 \mathrm{M})$ was mixed with $2.5 \mathrm{mLdeionized} \mathrm{water} \mathrm{and} 2.5 \mathrm{~mL} \mathrm{HAuCl}_{4}(0.001 \mathrm{M})$ under constant stirring. After that, $0.6 \mathrm{mLof}$ ice-cold sodium borohydride $(0.01 \mathrm{M})$ was added dropwise with continuous stirring until forming a brownish yellow solution. After stirring the solution for 2 minutes at $28^{\circ} \mathrm{C}$, the seed solution was prepared. The seed solution had to be used within 2-5 hours. Furthermore, $50 \mathrm{~mL}$ of CTAB solution $(0.2 \mathrm{M})$ was blended with $50 \mathrm{~mL} \mathrm{HAuCl}_{4}$ solution $(0.001 \mathrm{M})$, then added $1 \mathrm{~mL}$ silver nitrate $(0.01 \mathrm{M})$, and $0.8 \mathrm{~mL}$ of $0.1 \mathrm{M}$ ascorbic acid was added dropwise after gentle mixing until the growth solution color changes from dark yellow to colorless. Finally, $200 \mu \mathrm{L}$ of seed solution was added. The growth medium was kept constantly stirring at $28^{\circ} \mathrm{C}$ overnight in the dark place. The prepared GNRs were collected via centrifugation $(11,000 \mathrm{rpm})$ for 30 minutes. Then, adequate deionized water was used to wash GNRs twice by centrifuging $(11,000 \mathrm{rpm})$. The bare GNRs were dissolved in $25 \mathrm{~mL}$ deionized water after purification.

\section{Synthesis of gold nanorods @ palladium (GNRs@Pd)}

A typical procedure for the growth of Pd shells is shown below: the as-prepared GNRs solutions $(4 \mathrm{~mL})$ were mixed with 2 mLof $0.1 \mathrm{M}$ cetyltrimethylammonium bromide (CTAB) aqueous solution containing $20 \mu \mathrm{L}$ of $0.1 \mathrm{M}$ ascorbic acid (AA); $425 \mu \mathrm{L}$ of $2 \mathrm{mM} \mathrm{PdCl}_{4}{ }^{2-}$ $\left(0.035 \mathrm{~g}\right.$ of $\mathrm{PdCl}_{2}$ was dissolved in $2 \mathrm{~mL}$ of $0.2 \mathrm{M}$ aqueous $\mathrm{HCl}$ solution and then diluted into $100 \mathrm{~mL}$ with deionized water) was added. The mixtures were then shaken vigorously and placed in a $30^{\circ} \mathrm{C}$ water bath. Within several hours, the color of the solution changed from brown to dark gray, suggesting the formation of a Pd shell. After 14 hours, the reaction was stopped by centrifuging (12,000 rpm for 10 minutes) the solution twice.

\section{Eye patch preparation}

We added $3 \mathrm{~g}$ gelatin to $10 \mathrm{~mL}$ GNRs@ Pd and formed a solution in a $50^{\circ} \mathrm{C}$ water bath. Then, in order to get the shape of the eye patch, we added geniping $(20 \mu \mathrm{L}, 0.1 \mathrm{M})$ to absorb the appropriate amount of solution in the model. After 5 minutes, we dropped about 3-4 drops of $\mathrm{FeCl}_{3}$ solution.

\section{Cell experiments}

All cell lines, including human retinal epithelial 293 cells and retinal precursor R28 cells, were purchased from the Chinese Academy of Sciences Cell Bank of Type Culture Collection (CBTCCCAS, Shanghai, China) and cultured according to the relevant specifications. The cells were cultured in DMEM or 1640 medium (Hyclone, Logan, UT) supplemented with $4.5 \mathrm{~g} / \mathrm{L}$ glucose, $10 \%$ fetal bovine 
serum (FBS) and 1\% glutamine. Cultures were maintained at $37^{\circ} \mathrm{C}$ in a humidified atmosphere containing $5 \% \mathrm{CO}_{2}$. The in vitro cell cytotoxicity was performed using cell counting kit-8 (CCK-8) (Solarbio, Beijing, China).

\section{Animal experiments}

Sprague-Dawley rats were perfused with $0.9 \%$ saline solution and then perfused with 4\% PFA in PBS. The skin of the back and the eyelids were separated and cryoprotected in a $30 \%$ sucrose solution and cut into $5 \mu \mathrm{m}$ sections and H\&E stained. Ethical statement: 5 Male Sprague-Dawley rats (2-3 months of age) were obtained from the center of Laboratory Animal Science of Nanchang University. The animals were maintained in room temperature $\left(20-22^{\circ} \mathrm{C}\right)$ and $40-50 \%$ humidity. Rats were provided with water and a 12-hour light/dark cycle. All animal experiments were performed in accordance with the ARVO Statement for the Use of Animals in Ophthalmic and Vision Research, and were approved and monitored by the Institutional Animal Care and Use Committee of Nanchang University (no. 2016NC-020-02), China.

\section{Human tests}

General information

We used prospective interventional research methods. In April 2018, we included 20 young volunteers at the Eye Hospital of Nanchang University, including 10 males and 10 females, aged from $18-30$ years, average $=23.38 \pm 2.73$ years old; the spherical degree is $0-5.00 \mathrm{D}$, and the astigmatism is $0-1.00$ D. Subject inclusion criteria: aged $\geq 18$ years; discontinued wearing contact lens for more than 1 month; no history of family hereditary eye disease; no ocular trauma, history of laser, or surgery; no signs of systemic immune disease and eye infection; No drugs affecting ocular surface function were used locally or whole body during the week; no abnormalities were observed in slit lamp microscope and ophthalmoscopy; best corrected visual acuity $\geq 1.0$; reference standard [6], ocular surface disease index (ocular) Surface disease index, OSDI) $\leq 12$ points. Exclusion criteria: patients with eye or other eye diseases; diseases such as cardiovascular and cerebrovascular, liver, kidney, and hematopoietic system; and mental patients. All experiments involving human beings were approved by the Ethics Committee of the Affiliated Eye Hospital and the Biomedical Committee of Translational Medicine of Nanchang University (No. YK/92/2016). All volunteers signed informed consent documents in written form in accordance with the principles of the Declaration of Helsinki before the human test. The person in Figure S1 also signed written informed consent for the publication of his image.

\section{Methods}

All examinations were performed by the same experienced physician. The examination was performed in a well-lit room and the subject was confirmed not to be exposed to any electronic products prior to the examination. Subjects were tested for tear height, noninvasive BUT (noninvasive tear BUT, NITBUT), conjunctival hyperemia, lipid layer analysis, meibomian gland imaging, corneal fluorescein staining, and intraocular pressure measurement; all tests were repeated three times and averaged. Then, in the full refractive correction state, volunteers watch the same video for 3 hours in front of a computer about $30 \mathrm{~cm}$ away from the computer screen, and the above tests are repeated immediately after the operation.

\section{Tear meniscus height}

The tear meniscus height of the lower part of the cornea was measured by using a Keratograph 5M noninvasive ocular surface analyzer (Oculus, Germany). Subjects placed the head position in front of the central point in front of the inspection according to the inspection requirements.

The detector was debugged to the tear river height measurement mode, and an image was obtained after the subject blinked. The random software caliper tool was used to measure the height of the tear river below the center of the cornea.

\section{Measurement of NITBUT}

The inspector adjusted the Keratograph 5M noninvasive ocular surface analyzer to the BUT measurement mode. The subject was blinking normally, then focused and blinked twice, until the ring of the Placidoring projecting onto the cornea ruptured, and the duration was recorded.

\section{Measurement of conjunctival congestion}

Focusing the Keratograph 5M noninvasive ocular surface analyzer to the clearest conjunctival vessels, after the subject blinks normally, the bulbar conjunctiva is fully exposed and data is collected. After analyzing with the Keratograph $5 \mathrm{M}$ self-contained analysis system, the conjunctival hyperemia score and the limbal congestion degree score were automatically displayed. 


\section{Schirmer test}

A $5 \mathrm{~mm} \times 35 \mathrm{~mm}$ filter paper, bent by $5 \mathrm{~mm}$ at the end, was placed in the conjunctival sac of the inner third of the lower eyelid. The subject closed their eyes, and the length of the filter paper wetted by tears was measured after 5 minutes. The result of the normal human Schirmer test is $10-15 \mathrm{~mm} / 5$ minutes; $<10 \mathrm{~mm}$ is low secretion; and $<5 \mathrm{~mm}$ is dry eye. The Schirmer test in rabbit eyes is similar to human eye operation.

\section{Tear film lipid layer measurement}

We focused the Keratograph 5M noninvasive ocular surface analyzer to the superficial lipid layer of the cornea. The lipid layer image is acquired using a video device, while the subject is blinking normally. The tear film lipid layer was evaluated according to the four aspects of lipid layer thickness, distribution, sharpness, and color brightness. The observation of the thickness, uniform distribution, sharpness, and color brightness of the lipid layer was judged by the examiner.

\section{Statistical analysis}

Statistical data were expressed as the means \pm SD. The experiments were conducted three or four times. Differences between mean values were analyzed with one-way analysis of variance (ANOVA) followed by Tukey test for multiple comparisons. The nonparametric analysis followed by independent sample Kruskal-Wallis test was applied to determine the level of significance, instead of a standard one-way ANOVA, when these data were from a suspected nonnormal population. Significance was considered for $P=0.05$ or $P=0.01$.

\section{Results and discussion Ingredients and use of the eye patch}

As it shown in Figure 1, this eye patch includes three functional layers. From the inside (skin) to the outside (surface) are $\mathrm{FeCl}_{3}$, GNRs @ Pd, and temperature sensitive ink, consecutively. $\mathrm{FeCl}_{3}$ provides adhesion to the eye patch. ${ }^{23} \mathrm{GNRs}$ (a) Pd, the core material, converts visible light to heat. In
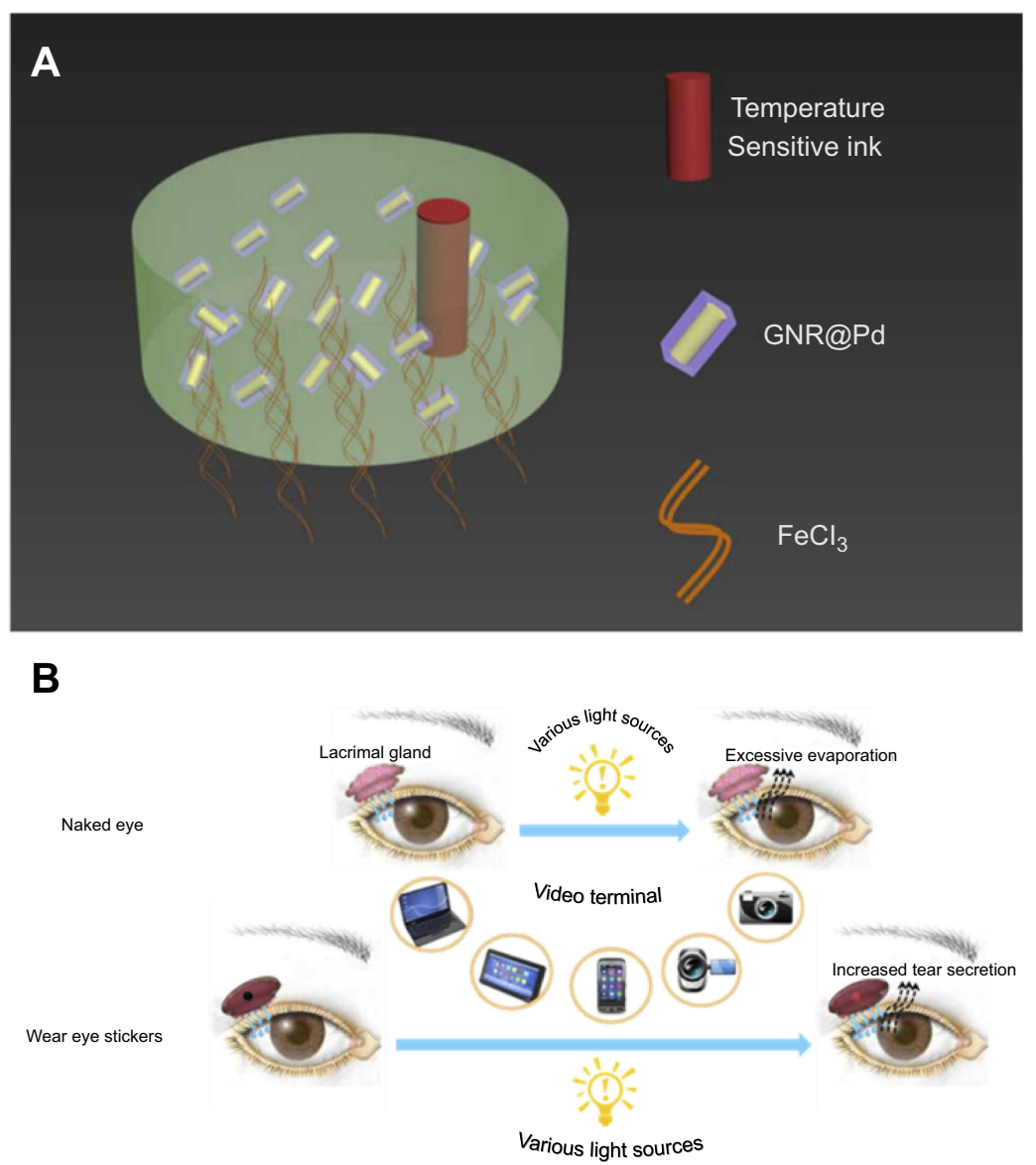

Figure I Ingredients and use of the eye patch. (A) The eye patch was composed of hydrogel, GNRs @ Pd, temperature sensitive ink, and FeCl3. (B) Schematic diagram of the function mechanism of the eye patch.

Abbreviation: GNRs @ Pd, gold nanorods @ palladium. 
addition, the built-in temperature sensitive ink (complex composed of amino resin \& styrene maleic anhydride copolymer) changes color based on patch temperature, serving as a warning to excessive eye usage. The lacrimal gland in normal eyes is located in the lacrimal fossa above the eyelid, which is the main organ for the secretion of tears. The proposed hydrogel eye patches can be attached to the surface of lacrimal gland. It can sense several visible lights and then heat up in response, which then stimulates the secretion of tears to moisture eyes.

\section{Characterization and heating performance of the eye patch}

Figure 2A shows the sticky effect of the hydrogel on human skin. Subsequent human trials showed that the eye patch was firmly adhered to the eyelid skin during 3 hours of videowatching. The video we provided also shows the stickiness of the eye patch (Video S1). Figure 2B is a scanning electron micrograph of the GNRs @ Pd nanorods. A scanning electron micrograph of Au is presented in Figure S2A. The corresponding energy spectrum data shows that the sample is a composite material of $\mathrm{Au}$ and $\mathrm{Pd}$ (Figure S2B). Figure $2 \mathrm{C}$ is the ultraviolet absorption spectrum of GNR and GNRs@ @ Pd. GNRs@ @ Pd had a broader characteristic absorption peak than pure gold nanorods. In order to verify the actual effect of illumination on smart eye patches, as shown in Figure 2D, white LED light was irradiated on the surface of smart eye patches, and the relative temperature change before and after irradiation is shown in Figures $2 \mathrm{E}$ and $\mathrm{F}$, respectively. We further explored the performance of the eye patch under different light sources. Figure $2 \mathrm{G}$ showed that, under white light (blue light + yellow light), the eye patch had the best heating effect. For monochromatic lights, the warming effect under blue light was better than that under yellow light. Since research has shown that shortwavelength blue light is more likely to cause ocular surface damage than long-wavelength yellow light, ${ }^{24}$ the proposed eye patch was in line with the daily eye protection needs. Moreover, under the same light irradiation, the warming effect of eye patch composed of GNRs @ Pd was better than that of gold nanorods or gold nanoparticles (Figure 2H). A complex amino resin and styrene maleic anhydride copolymer was implanted in the eye patch. After illumination, changes in eye patch temperature consequently induced color change of the temperature sensitive ink (Figures $2 \mathrm{I}$ and $\mathrm{J}$ ). In order to further verify the stability of the eye patch, an intermittent light experiment was performed on a constant temperature heating plate $\left(37^{\circ} \mathrm{C}\right)$. The eye patch effectively heated up, and its
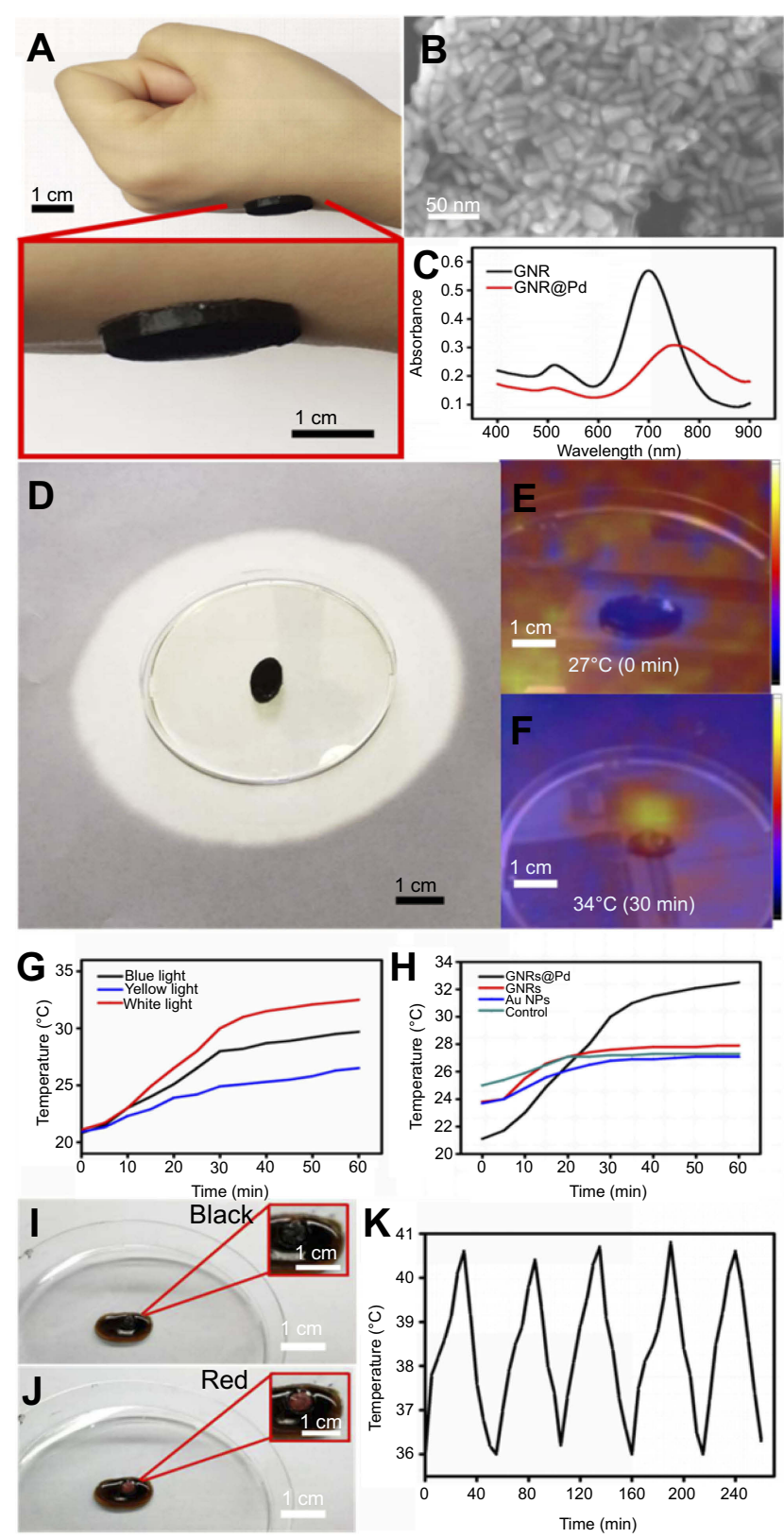

Figure 2 The characterization and heating performance of the eye patch. (A) The Sticky effect of eye patch on human skin. (B) Scanning electron micrograph diagram of GNRs @ Pd. (C) Ultraviolet absorption spectrum of GNR, GNRs @ Pd. (D) An experimental picture of the eye patch under light-illuminating. (E, F) Infrared temperature profile before and after illumination. (G) The temperature rise diagram of the eye patch under different color light illumination. $(\mathbf{H})$ The temperature rise diagram of the eye patch made of GNRs @ Pd, gold nanorods, or gold nanoparticles. (I, J) After the temperature-sensitive ink was implanted, the color change map of the multi-functional eye patch before and after illumination. (K) Temperature elevation of the eye patch over five irradiation cycles.

Abbreviation: GNRs@ @ Pd, gold nanorods @ palladium.

temperature stabilized at around $41^{\circ} \mathrm{C}$ (Figure 2K), which thus offered an appropriately gentle heating source to stimulate the lacrimal glands without burning the skin. More importantly, this eye patch could be repeatedly used according to the cycles of illumination. 

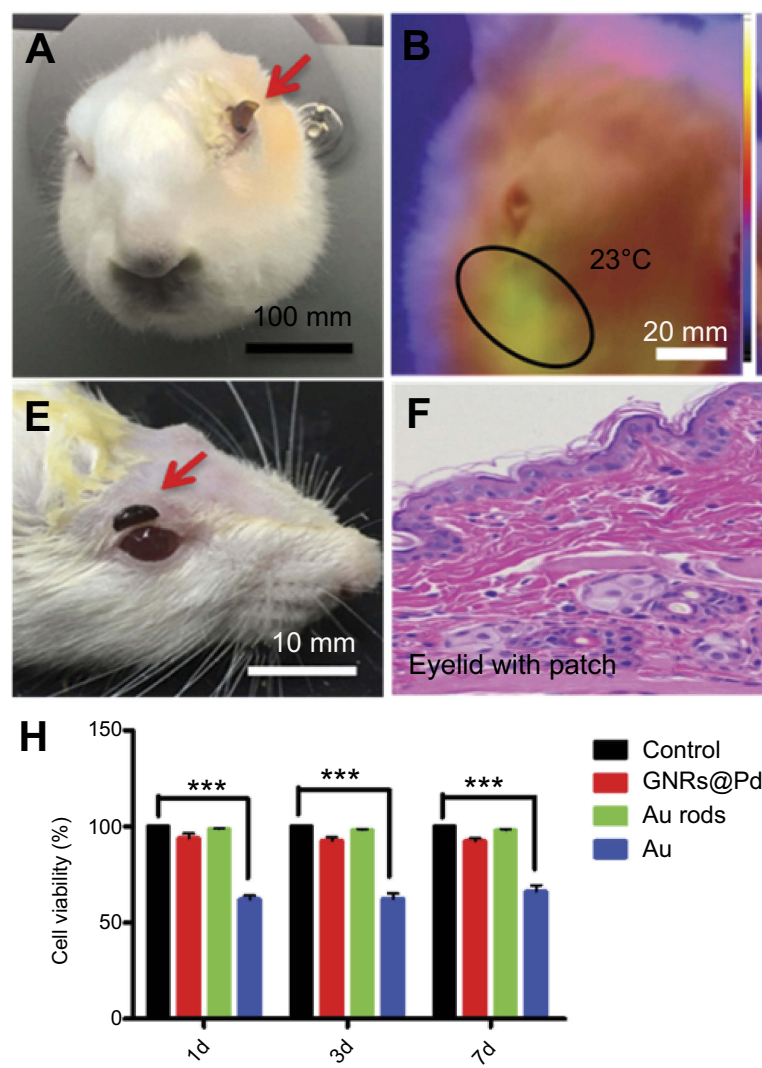
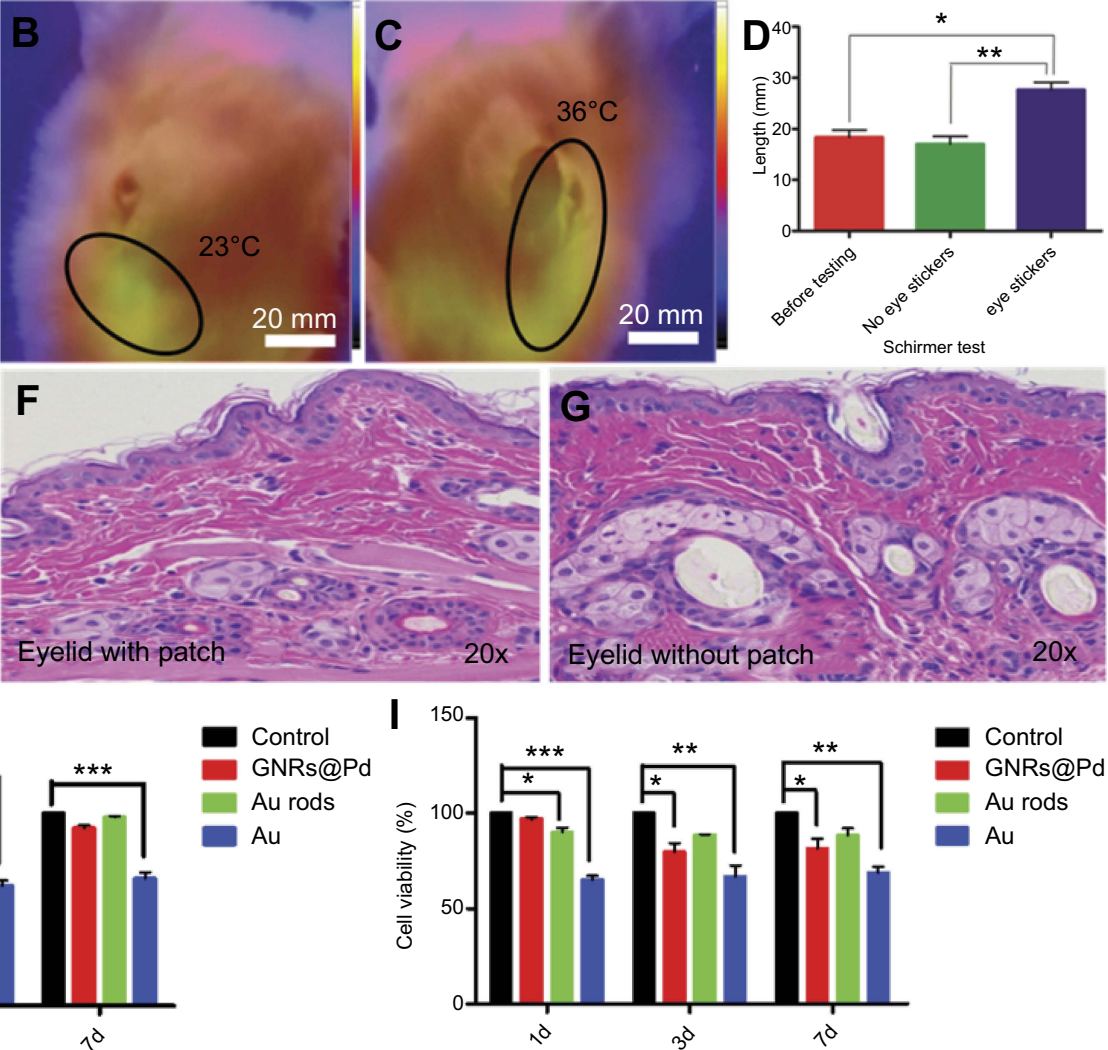

Figure 3 The safety assessment of the eye patch. (A) A photo of a rabbit pasted with an eye patch. (B, C) The infrared heat distribution maps of the rabbit eye with or without the eye patch. (D) The tear secretion test of rabbit eyes. (E) The picture of the mouse paste eye patch. (F, G) The HE staining of the mouse's eyelid skin without or with the eye patch for 24 hours. (H) The cytotoxicity tests by using 293 cells. (I) The cytotoxicity tests by using R28 cells. Each group contains four samples, Error bars: SEM. Unpaired $t$-test, $* P<0.05, * * P<0.01, * * * P<0.001$.

Abbreviations: HE, hematoxylin-eosin; GNRs@Pd, gold nanorods @ palladium.

\section{Safety assessment of the eye patch}

Figure $3 \mathrm{~A}$ shows the attached eye patch on the rabbit eye. Figures $3 \mathrm{~B}$ and $\mathrm{C}$ were the infrared heat distribution maps of the rabbit eye with or without the eye patch. The results showed that the temperature on the side with eye patch was significantly higher than the side without the eye patch. A tear secretion test of rabbit eyes in Figure 3D showed that the tear secretion was significantly increased in the rabbit eyes with eye patch. The relative Schirmer test was presented in the Supplementary materials (Figure $\mathrm{S} 1 \mathrm{~A})$. Figure $3 \mathrm{E}$ was a picture of the attached eye patch on a rat eye. Figures $3 F$ and $G$ were $H \& E$ staining of the mouse's eyelid skin with or without eye patch, no significant differences were found in the results. We also performed H\&E staining on the dorsal skin of mice with or without eye patches, and similar results were also obtained (Figure S3). This showed that the eye patch had no obvious toxic side-effects on the skin. Figure $3 \mathrm{H}$ was a cytotoxicity test of 293 cells (human renal epithelial cell line). The cells were cultured with Au rod, GNRs @
Pd, Au-soaked medium for 1 day, 3 days, and 7 days, respectively. The results showthat there was no obvious cytotoxicity in Au rod and GNRs @ Pd, but the cytotoxicity of Au was statistically significant $\left({ }^{*} P<0.05\right)$. Figure $3 \mathrm{I}$ was a cytotoxicity test of R28 cells (retinal precursor cell line). As a result, Au rods and GNRs@ Pd had slight cytotoxicity, but still less than bare gold. These experimental data demonstrated the biosafety of the proposed eye patch. In practical usage, a small amount of this hydrogel would not cause significant side-effects, even if it flows into the eye accidently.

\section{Customization of eye patches and evaluation of dry eye indicators}

In order to meet the needs of different human eye structures, the shapes of eye patches could be customized by 3D printing using different eye patch models. Corresponding types of eye patches were obtained. As shown in Figures S4A-S4D, they were customized 


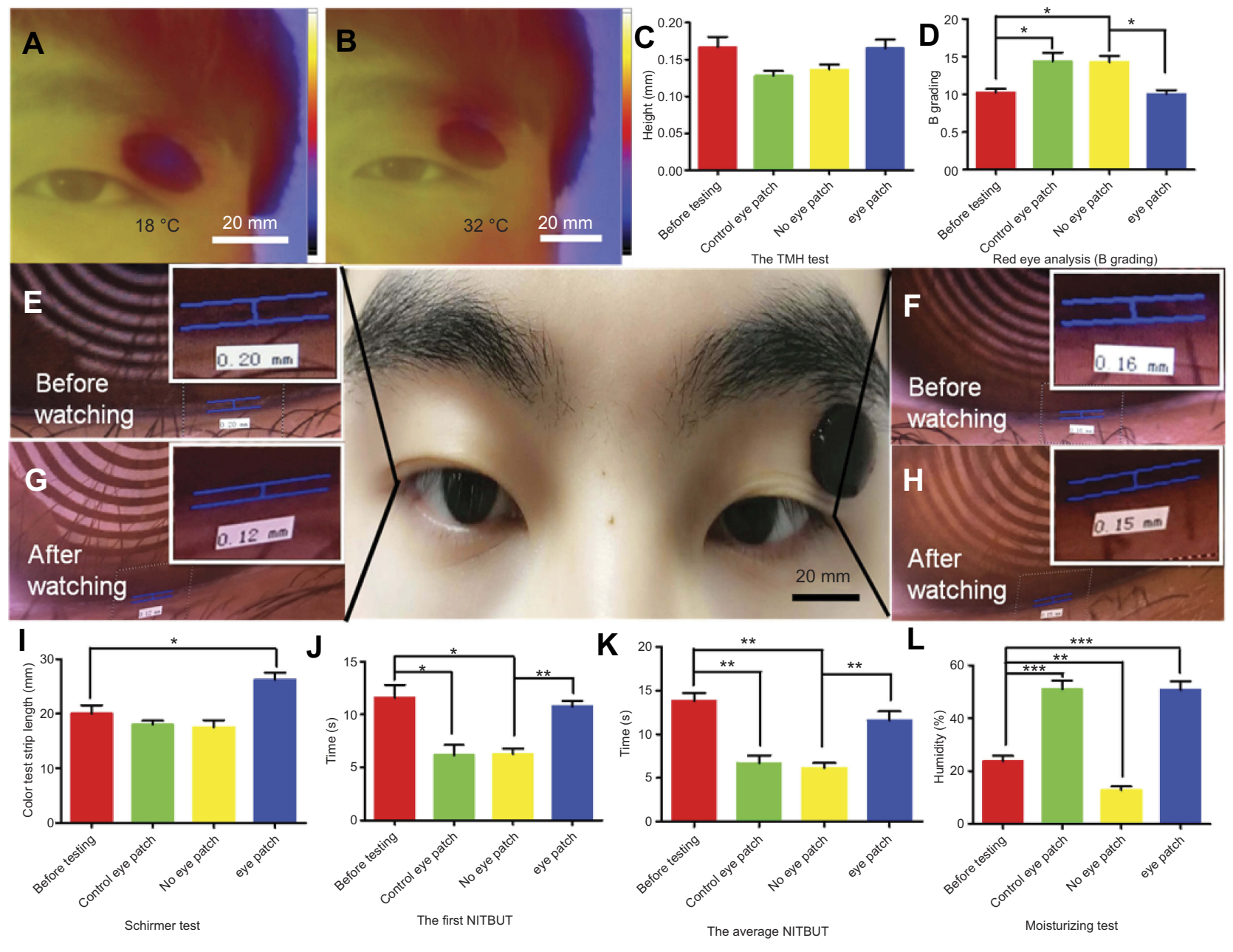

Figure 4 Customization of eye patches and evaluation of dry eye indicators. (A, B) The infrared heat distribution maps of the human with pasted eye patch before and after illumination. (C, D) TMH and red eye analysis (B Grading) of the human eye. (E, G) Photos of TMH of the groups without eye patches before and after watching the video for 3 hours. (F, H) Photos of TMH of the groups with eye patches before and after watching the same video. (I) The tear secretion test of human eyes was performed before or after watching a 3 hour video. (J) The first NITBUT test of human eyes was performed before or after watching a 3 hour video. (K) The average NITBUT test of human eyes was performed before or after watching a 3 hour video. (L) The eye patch moisturizing test was performed before or after watching a 3 hour video. Each group contained ten eyes. Error bars: SEM (unpaired $t$-test, $* P<0.05, * * P<0.01$, $* * * P<0.00$ I).

Abbreviations: TMH, tear meniscus height; NITBUT, noninvasive tear breakup time.

elliptical, pentagonal, crescent, and circular 3D models. Figures S5A-S5D are photos of the corresponding eye patch. Figures S6A-S6D are photos of humans with different eye patches.

Finally, we conducted a series of human experiments to test the effectiveness of the eye patch. Figures $4 \mathrm{~A}$ and $\mathrm{B}$ are infrared heat distribution maps of the pasted eye patch on human eyes before and after illumination, the temperature of the eye patch after light exposure was significantly higher than that before light exposure, which indicated that the eye patch was stimulated and warmed up. To further confirm the effectiveness of this eye patch in practice, several volunteers watched the same video on the computer for 3 hours. The volunteers attached the experimental eye patch on the right eye and a control eye patch that did not have heating properties on the left eye. Figures $4 \mathrm{C}$ and $\mathrm{D}$ showthe results of tear meniscus height (TMH) and the red eye analysis (B Grading) of the human eyes. After watching the video, the TMH of the group without eye patches and the group with control eye patches reduced, but the TMH of the group with eye patches increased. The result of the red eye analysis (B Grade) showed that the value of the group without eye patches and the group with control eye patches increased, while this harmful value was reduced by using the experimental eye patch. Figures $4 \mathrm{E}-4 \mathrm{H}$ are photos of the TMH before and after watching the video. Figure 4I shows that the tear secretion in the eye patch group was significantly increased compared to the control group, and to the non-eye patch group. Figure 4J 
showsthat the first NITBUT of the group without eye patches and the group with control eye patches were significantly lower than the eye patch group. Figure $4 \mathrm{~K}$ showsthat the result of the average NITBUT was consistent with the first NITBUT. In view of the significant effect of lipid layers on tear film stability, we supplemented the test for lipid layers in subjects (Figure S7). The results show that watching the video for 3 hours did not affect the lipid layer of the subject, and our eye patch also hardly affected the lipid layer. This may be due to the fact that we were attaching the eye patch to the lacrimal gland rather than the meibomian glands (the main glands that produce the lipid layer). These tests were measured with the Keratograph 5M noninvasive ocular surface analyzer (Figures S1B and S1C). Tear meniscus are the fluid arcs formed by tears between the eyelid and the cornea or the bulbar conjunctiva. TMH reflects tear secretion. ${ }^{25}$ The red eye analysis is one of the important indicators for the diagnosis of ocular surface diseases. ${ }^{26}$ NITBUT is the main indicator which reflects the stability of tear film. The above items are the main indicators for evaluating and diagnosing dry eye syndrome, the results demonstrated that the hydrogel eye patch that we developed could indeed alleviate dry eye.

In addition to testing the effect of the eye patch on dry eye, we also tested the effect of the eye patch itself on the skin. Figure 4 shows an eye patch moisturizing test, the moisture content of the eyelid skin (with or without eye patch) after watching the video for 3 hours was evaluated. We found that, compared to the control group (before watching the video), the eye patch significantly increased skin moisture. This result shows that the eye patch can not only relieve dry eye but also moisturize the eyelid skin.

\section{Conclusion}

In this paper, we propose direct, energy-saving, convenient, and relatively safe (without the use of preservativecontaining artificial tears) eye-care materials to deal with the worldwide problem of dry eye. To the best of our knowledge, this is the first time that hydrogel composites with visible light response have been used in dry eyerelated research. Corresponding animal and human experiments have demonstrated the safety and effectiveness of our proposed concept. In follow-up experiments, the longterm safety of this eye patch is still our first priority. In addition, since the prepared eye patch can effectively and frequently stimulate the lacrimal gland secretion, whether this strategy will affect the normal secretion of the lacrimal gland is also a potential concern. Moreover, given that dry eye with increased evaporation of the tear film accounts for a significant proportion of the total dry eye population, ${ }^{27}$ the improvement of the lipid layer, which is closely related to tear film evaporation, is also a key issue for our future work. In the future, more systematic works are still needed to optimize the structure and contents of this promising material.

\section{Acknowledgments}

This work was supported by the National Natural Science Foundation of China (No. 31860263 and 21461015 to Xiaolei Wang; No. 81271425,81860170 and 81260148 to $\mathrm{Xu}$ Zhang); and the Science Foundation of Jiangxi Provincial Department of Education (20165BCB19002, KJLD14010, 20153BCB23035, and 20161ACB21002 to Xiaolei Wang; 20151BBG70243 and 20181ACG70010 to Xu Zhang).

\section{Disclosure}

The authors report no conflicts of interest in this work.

\section{References}

1. Shimazaki-Den S, Dogru M, Higa K, Shimazaki J. Symptoms, visual function, and mucin expression of eyes with tear film instability. Cornea. 2013;32(9):1211-1218. doi:10.1097/ICO.0b013e318295a2a5

2. Yamamoto Y, Yokoi N, Higashihara H, et al. Clinical characteristics of short tear film breakup time (but) -type dry eye. Nihon Ganka Gakkai Zasshi. 2012;116(12):1137-1143.

3. Himebaugh NL, Begley CG, Bradley A, Wilkinson JA. Blinking and tear break-up during four visual tasks. Optom Vis Sci. 2009;86(2): E106-E114. doi:10.1097/OPX.0b013e318194e962

4. Messmer EM. The pathophysiology, diagnosis, and treatment of dry eye disease. Dtsch Arztebl Int. 2015;112(5):71-81, 82. doi:10.3238/ arztebl.2015.0071

5. Lemp MA, Crews LA, Bron AJ, Foulks GN, Sullivan BD. Distribution of aqueous-deficient and evaporative dry eye in a clinic-based patient cohort: a retrospective study. Cornea. 2012;31 (5):472. doi:10.1097/ICO.0b013e318225415a

6. Heiligenhaus A, Koch JM, Kruse FE, Schwarz C, Waubke TN. Diagnosis and and differentiation of dry eye disorders. Ophthalmologe. 1995;92(1):6-11.

7. Tong L, Chaurasia SS, Mehta JS, Beuerman RW. Screening for meibomian gland disease: its relation to dry eye subtypes and symptoms in a tertiary referral clinic in singapore. Invest Ophth Vis Sci. 2010;51(7):3449. doi:10.1167/iovs.09-4445

8. Baudouin C, Labbe A, Liang H, Pauly A, Brignole-Baudouin F. Preservatives in eyedrops: the good, the bad and the ugly. Prog Retin Eye Res. 2010;29(4):312-334. doi:10.1016/j.preteyeres.2010.03.001

9. Baudouin C, Denoyer A, Desbenoit N, Hamm G, Grise A. In vitro and in vivo experimental studies on trabecular meshwork degeneration induced by benzalkonium chloride (an American Ophthalmological Society thesis). Trans Am Ophthalmol Soc. 2012;110:40-63.

10. Zha Z, Yue X, Ren Q, Dai Z. Uniform polypyrrole nanoparticles with high photothermal conversion efficiency for photothermal ablation of cancer cells. Adv Mater. 2013;25(5):777-782. doi:10.1002/ adma.201202211

11. Zhu XM, Wan HY, Jia H, Liu L, Wang J. Porous pt nanoparticles with high Near-Infrared photothermal conversion efficiencies for photothermal therapy. Adv Healthc Mater. 2016;5(24):3165. doi:10.1002/adhm.201601058 
12. Wu B, Wan B, Lu ST, et al. Near-infrared light-triggered theranostics for tumor-specific enhanced multimodal imaging and photothermal therapy. Int J Nanomed. 2017;12:4467-4478. doi:10.2147/IJN.S137835

13. Tian N, Zhou ZY, Sun SG. Electrochemical preparation of Pd nanorods with high-index facets. Chem Commun. 2009;12(12):1502-1504. doi:10.1039/b819751b

14. Carbóargibay E, Rodríguezgonzález B, Gómezgraña S, et al. The crystalline structure of gold nanorods revisited: evidence for higher-index lateral facets. Angew Chem Int Ed. 2010;122 (49):9587-9590. doi:10.1002/ange.201004910

15. Zhu J, Yong KT, Roy I, et al. Additive controlled synthesis of gold nanorods (GNRs) for two-photon luminescence imaging of cancer cells. Nanotechnology. 2010;21(28):285106. doi:10.1088/0957-4484/ 21/28/285106

16. Chen WH, Yang CX, Qiu WX, et al. Multifunctional theranostic nanoplatform for cancer combined therapy based on gold nanorods. Adv Healthc Mater. 2015;4(15):2247-2259. doi:10.1002/adhm.201500453

17. Liu Y, Xu M, Chen Q, et al. Gold nanorods/mesoporous silica-based nanocomposite as theranostic agents for targeting near-infrared imaging and photothermal therapy induced with laser. Int J Nanomed. 2015;10:4747-4761. doi:10.2147/IJN.S82940

18. Kalmodia S, Harjwani J, Rajeswari R, et al. Synthesis and characterization of surface-enhanced Raman-scattered gold nanoparticles. Int J Nanomedicine. 2013;2013(Issue 1):4327-4338. doi:10.2147/ IJN.S49447

19. Fan Z, Zhu Y, Huang X, et al. Synthesis of ultrathin Face-CenteredCubic au@pt and au@pd Core-Shell nanoplates from Hexagonal-Close-Packed au square sheets. Angewandte Chemie. 2015;54(19):5672-5676. doi:10.1002/anie.201500993
20. Zhao X, Lang Q, Yildirimer L, et al. Photocrosslinkable gelatin hydrogel for epidermal tissue engineering. Adv Healthc Mater. 2016;5(1):108-118. doi:10.1002/adhm.201500005

21. Ge L, Li Q, Jiang J, et al. Integration of nondegradable polystyrene and degradable gelatin in a core-sheath nanofibrous patch for pelvic reconstruction. Int J Nanomed. 2015;10:3193-3201. doi:10.2147/IJN. S75802

22. Kirchmajer D, Watson C, Ranson M, Panhuis M. Gelapin, a degradable genipin cross-linked gelatin hydrogel. RSC $A d v$. 2012;3(4):1073-1081. doi:10.1039/C2RA22859A

23. Siglreitmeier M, Wu B, Kollmann T, et al. Multifunctional layered magnetic composites. Beilstein J Nanotechnol. 2015;6(1):134-148. doi:10.3762/bjnano.6.13

24. Lee HS, Cui L, Li Y, et al. Influence of light emitting Diode-Derived blue light overexposure on mouse ocular surface. PLoS One. 2016;11 (8):e161041. doi:10.1371/journal.pone.0161041

25. Doughty MJ, Laiquzzaman M, Oblak E, Button N. The tear (lacrimal) meniscus height in human eyes: a useful clinical measure or an unusable variable sign? Cont Lens Anterior Eye. 2002;25 (2):57-65

26. Rodriguez JD, Johnston PR, Ousler GR, Smith LM, Abelson MB Automated grading system for evaluation of ocular redness associated with dry eye. Clin Ophthalmol. 2013;7:1197-1204. doi:10.2147/OPTH.S39703

27. Craig JP, Nelson JD, Azar DT, et al. TFOS DEWS II report executive summary. Ocul Surf. 2017;15(4):802. doi:10.1016/j. jtos.2017.08.003
International Journal of Nanomedicine

\section{Publish your work in this journal}

The International Journal of Nanomedicine is an international, peerreviewed journal focusing on the application of nanotechnology in diagnostics, therapeutics, and drug delivery systems throughout the biomedical field. This journal is indexed on PubMed Central, MedLine, CAS, SciSearch ${ }^{\circledR}$, Current Contents ${ }^{\mathbb{R}} /$ Clinical Medicine,

\section{Dovepress}

Journal Citation Reports/Science Edition, EMBase, Scopus and the Elsevier Bibliographic databases. The manuscript management system is completely online and includes a very quick and fair peer-review system, which is all easy to use. Visit http://www.dovepress.com/ testimonials.php to read real quotes from published authors. 